\title{
VIRTUAL SIMULATION AND MULTI BODY DYNAMICS ANALYSIS OF COCONUT DEHUSKING MACHINE
}

\author{
Rajashekar M ${ }^{1}$, S.Mohankumar ${ }^{2}$ \\ ${ }^{1}$ Research Scholar, Mechanical Engineering Department, MCE, Hassan, VTU, Belgaum, India \\ ${ }^{2}$ Professor, Malnad College of Engineering, Hassan, VTU, Belgaum, India
}

\begin{abstract}
The coconut dehusking machine is finding a major application in our country. The available methods to dehusk the coconut are manual, semi automatic and with the help of fully automatic machines. Coconut dehusking with machete requires more skill. Need for the improvement in present method is the lack of sufficient manpower. Traditional devices currently in use, such as the blade and spear are difficult and less productive. Even though limited number of automated machines was available, the reasons for the failure of these machines include unsatisfactory and incomplete dehusking, breakage of the coconut shell while dehusking, spoilage of useful coir, greater effort needed than manual methods, etc. Based on this realization the necessity of a mechanism which simplify as well as increases the productivity of the coconut dehusking. Several trials are made so far in the manufacture of coconut dehusking machines. But they are not successful and effective in replacing man handling tools. Hence by observing the pitfalls of manual dehusking tools, and existing automated dehusker, the concept of dehusking of coconut machine is simulated in the virtual environment.

In this paper, the virtual prototype system framework of the coconut dehusking machine is simulated, and it consists of parts like main and feeding pipe, blades, helical spring and a collar. A conceptual design of the three-dimension (3-D) model of the dehusking machine is designed using CAD tool SOLIDWORKS 2010 and its Multi-Body Dynamic simulation is carried out by means of MSC ADAMS View 2012 software. The results are intended to show the forces and torques acting on the blades and piston during dehusking operation this gives scope for improvising the virtual prototype and hence is beneficial from point of view of modification of conceptual model and arriving at the final prototype.
\end{abstract}

Keywords: CAD, Simulation, Design, Solid Works ADAMS, Multi Body Dynamics, Coconut Dehusking Machine $* * *$

\section{INTRODUCTION}

Our country has recently became one of the top coconut producer, about seventeen billion in worldwide. Coconut is being cultivated in an area of over two million hectares in India. The estimated coconut seeds produced in our country about thirty million per year [4]. Removing husk of coconut is one of the major post harvest operation, which is required for making the coconut by products. The coconut husk is raw material for coir production companies.

Now a days due to participation of government and other financial institution, the growth of industries especially coconut industries such as coconut powder making industries, coir making industries are given wider scope. The quantity of coconut required by one such single plant may vary from 10,000 to 40,000 coconuts per day. In such cases dehusking of coconut by conventional methods becomes very difficult and uneconomical, "Virtual Prototyping of Coconut De-husking Machine" unless mechanical means whose productivity is much longer than that of conventional methods are adopted to meet all these requirements. So to meet such requirements the coconut dehusking machine has to be designed, modelled and simulated .And the simulation of the machine in virtual environment will ensure the proper working of the dehusking machine.
The configured Virtual model of the Coconut Dehusking Machine is modelled using SOLIDWORKS 2010 modelling software. Then it is imported into the ADAMS VIEW, and by applying necessary joints, motions and contacts the model is simulated to study its Dynamic Behaviour.

\section{METHODS AND MATERIALS}

Coconut (cocos nucifera) is one of the world most useful and important perennial plants [7]. A large Coconut shape, is up to $290 \mathrm{~mm}$ long and $240 \mathrm{~mm}$ wide, an individual coconut fruit shown in Figure 1.a is built up with a outer thick fibrous husk, coconut fruit ,milk covered by hard core shell.

Coconut size varies from 147 to $196 \mathrm{~mm}$ in lateral dimension and 245 to $294 \mathrm{~mm}$ along its length At the top end of coconut there are 3 holes of medium soft material similar to eyes on head Inner core consist of thin, white, layer, about 12 to $15 \mathrm{~mm}$ thick at maturity, known as the "coconut fruit". The interior of the coconut is partially filled with a milky liquid and partially raw fruit. The outside surface of the nut is smooth with pale yellow or pale green colour The thickness of outside husk is $25 \mathrm{~mm}$ to $50 \mathrm{~mm}$. The inner core of nut is very hard with brown colour which surrounds coconut fruit. Coconut husks are the rough which has to be removed for the usage of coconut. 


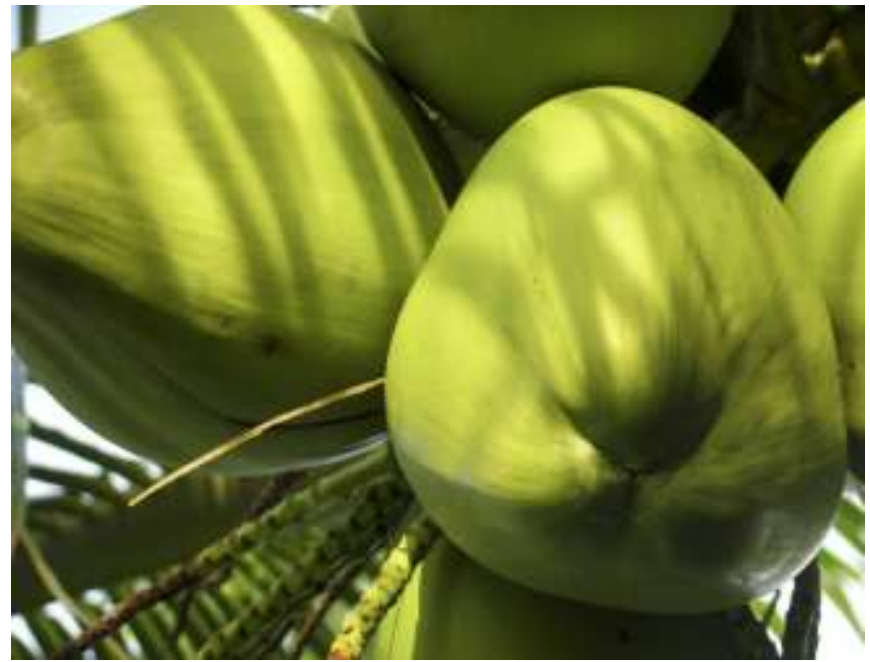

Fig 1.a Structure of Coconut

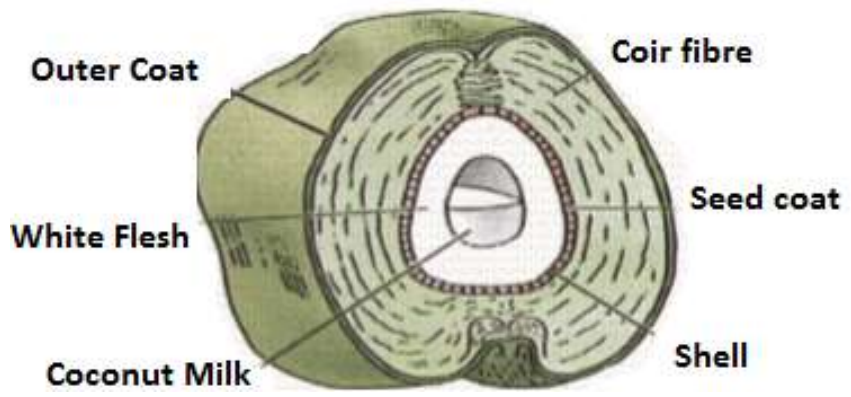

Fig 1.b Structure of Coconut

\subsection{Methodology Adopted for Conceptual Design}

In general equipment/machinery fabrication industries, CAD technology has been very widely applied to various fields. But Farm machinery still remains an the primary stage, which based on hand work such as objects, models and drawings and samples to complete the whole process of Farm machinery body design method without using the modern CAD design software tools Rajashekar M [9]. At present, foreign farm machinery companies have started to use CAD modern technology, while problems such as not precise enough, long design cycle still exist in domestic agricultural machinery companies.

Design of any machine is not so simple. Over a period of time, design of different machines was done by using the paper and drafting tools, but now most of the designing work is done by using CAD tools. In comparison with traditional prototyping method, Virtual Simulation Design provides tremendous time and cost savings in making fully functioning physical model with the aid of CAD technologies.

The size of the coconut varies and accordingly they can be classified into three groups [6].

1) Maximum size $[\mathrm{D}=200-230 \mathrm{~mm} ; \mathrm{L}=180-220 \mathrm{~mm}]$

2) Medium size $[\mathrm{D}=150-200 \mathrm{~mm} ; \mathrm{L}=170-190 \mathrm{~mm}]$

3) Minimum size $[\mathrm{D}=120-150 \mathrm{~mm} ; \mathrm{L}=150-180 \mathrm{~mm}]$
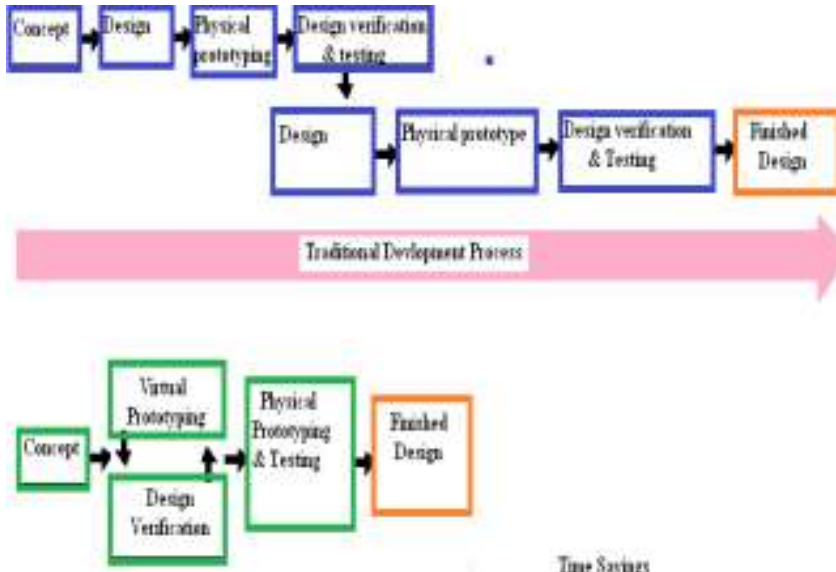

Time \$asąs

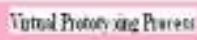

Fig 2.Traditional and Virtual Prototype Design

The principle adopted in dehusking machine is much similar to manual process the blades are given a bend at the surface and also with their base thickness decreases gradually, Dehusking takes place quickly and completely.

Depending on the simplifications in design, number of coconuts dehusked and efficiency the models are as below.

Design 1: In this machine the coconut is placed in between two roller drums rotating at slightly in different speed. The spikes on the periphery of the drums pierce in to the husk due to twisting action which results in removal of husk from coconut.

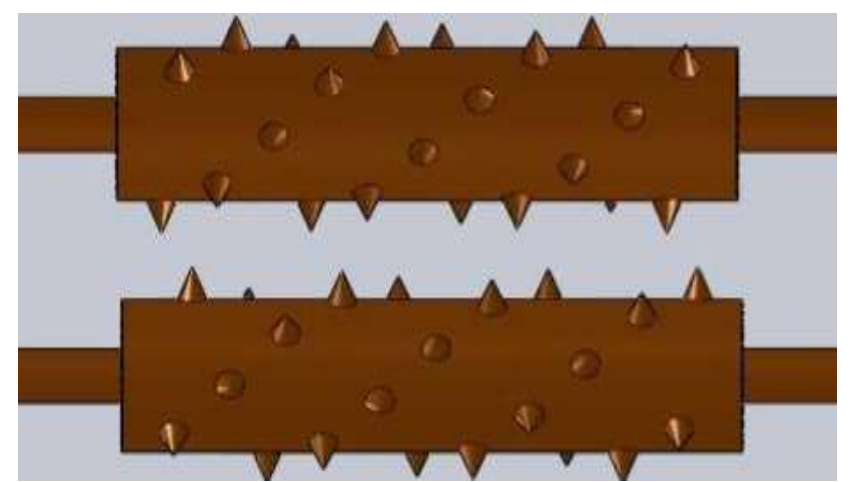

Fig 3: Virtual Design of Roller Drum mechanism

Design 2: In this Design the coconut is allowed to drop in vertical position in the coconut holder, and the blades at the top with a force are applied such that it pierces into the coconut. After piercing of blades a twisting force is applied which peels off the husk from coconut. From literature survey and by verifying it virtually both the models it is found that the Design 2 has better efficiency of dehusking in removing off the husk from coconut completely. By considering the drawbacks of the present dehusking machine is modified to meet the following requirements.

$>$ Skill of conventional operation is to be substituted.

$>$ Developing simple mechanism with less labor

$>$ Danger to the worker be eliminated. 
$>$ Effective feeding and positioning of coconut

$>$ Provisions to retain the spout on the coconut.

$>$ To Dehusk both dry and wet coconut efficiently

$>$ Eliminating the breakage of coconut shell.

$>$ Cost of the machine should be less.

$>$ Output must be more with the conventional Dehusking.

The important design parameters to arrive at final dimensions and shape of the conceptual dehusking machine for effective dehusking is discussed here. The shape of the cutting blades for effective dehusking and feeding facility to allow one coconut at a time is provided.The principle adopted in dehusking machine is very much similar to the manual process. The blades are given a bend at the bottom and also with their cutting edge's thickness increases from top gradually, dehusking takes place quickly and completely. To accommodate the piston and blades the main pipe is necessary. The main pipe must be selected such that only one coconut should position in the pipe. It should not allow the coconut to incline or fall transversely over the blades. To meet these needs, the size of the pipe selected is equal to the maximum size of the coconuts available, i.e., $230 \mathrm{~mm}$ inside dia. Now, in this pipe the coconuts can position themselves longitudinally. They cannot fall diameter wise, since the length of the medium sized coconuts is 170 to $190 \mathrm{~mm}$ which is slightly less than the diameter of the pipe. The length of the main pipe required is decided by the interference of the piston and connecting rod play over the sides of the pipe. By considering the maximum length of the coconut the stroke length taken as $300 \mathrm{~mm}$. The pipe should accommodate the position of the blade tip which is $30 \mathrm{~mm}$ below it and coconut of $220 \mathrm{~mm}$ length. Thus the total length of the pipe is equal to,

The operating and feeding system $=250 \mathrm{~mm}$

Piston length $=170 \mathrm{~mm}$

Gap above the blade is $=30 \mathrm{~mm}$

Total Height of the cylinder $=250+170+30=450 \mathrm{~mm}$

Inner diameter of cylinder $=230 \mathrm{~mm}$

Outer diameter of cylinder $=240 \mathrm{~mm}$

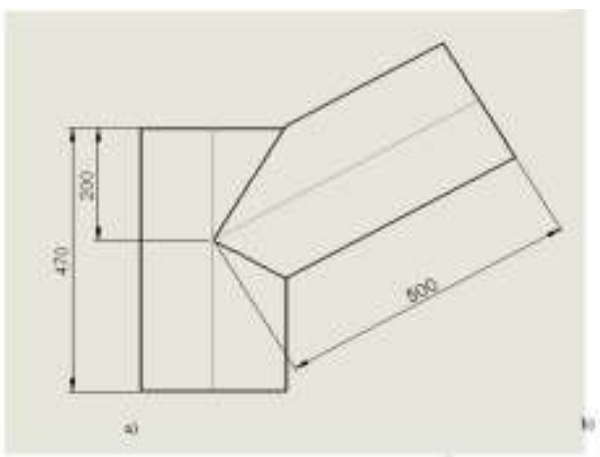

Fig 4 Main and Feeding pipe a) Top view

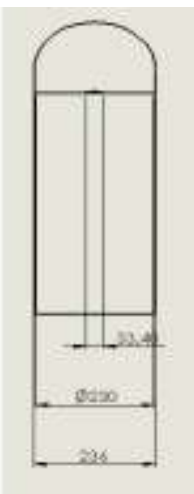

b) side view

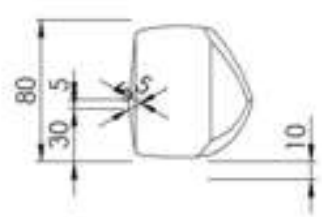

a)
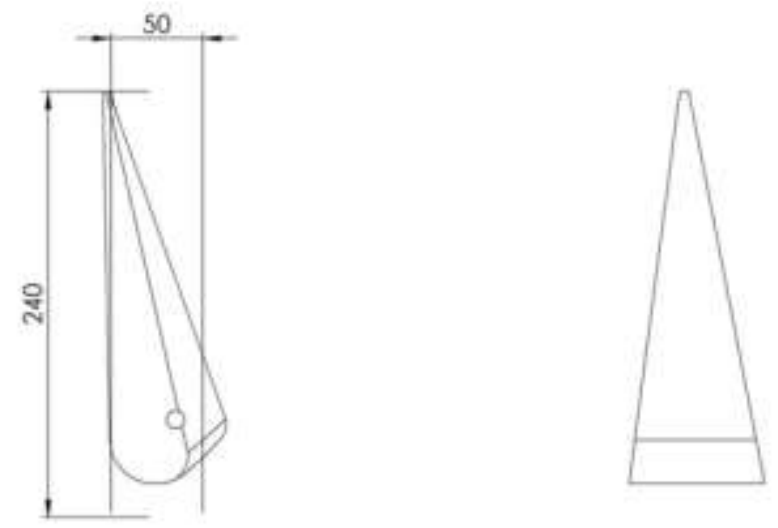

b)

c)

Fig 5 Blade a) Top view b) Side view c) Front view

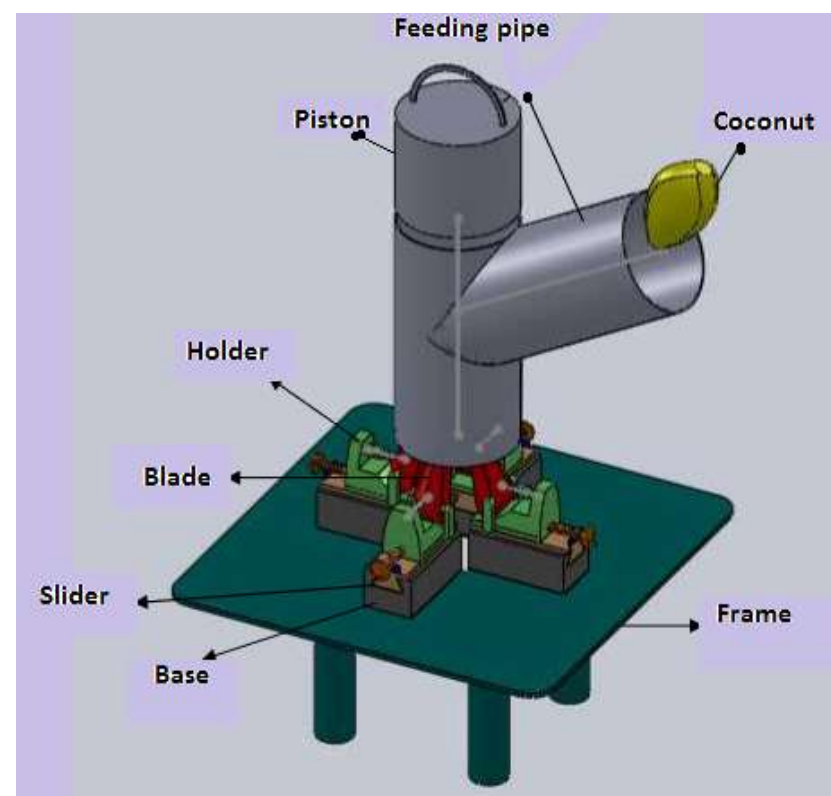

Fig 5 Virtual Coconut Dehusking Machine

Feeding mechanism consists of main pipe, feeding pipe and hopper. Main pipe is having hollow cylindrical shape.

The piston moves inside the main pipe and also coconut falls through this. Main pipe is placed just above the cutting blades such that the coconut gets placed at the required position on blades as shown in assembly. Feeding pipe is also designed same as that of main pipe and is inclined with the main pipe at an angle of $30^{\circ}$. At this particular angle the coconut slides over feeding pipe as required for the dehusking process. Hopper is conical in shape and is narrowed at the feeding pipe end. There are four blades used to dehusk the coconut. This will give the efficient dehusking. The shape and sizes of blades are given in next chapter. The 
blades are placed such that only the husk of coconut gets peeled off without damaging the shell of coconut. The position of the blades with respect to each other is made by using the trial and error method in the virtual environment so that to achieve proper dehusking of coconut.

In the coconut, one end is called embryonic end and the another end is called ridge end. The thickness of the husk at the ridge end is less as compared to embryonic end. Also piercing and removing should be done at the embryonic end to reduce the force required. Hence by experiment [6] it was found that force of about $600 \mathrm{Kg}$ was to be exerted on the coconut for dehusking and hence a power of $0.75 \mathrm{H} . \mathrm{P}$ is required. In this model a motor of 1 H.P is assumed whose final speed of $10 \mathrm{rpm}$ is assumed and with a feed rate of 10 coconuts per minute [6]. Spring steel is used for blades since it gives hardness and retains the keen edges for long time. The blade height is sufficiently long with the gradual increase in its cutting edge thickness, to affect the efficient peeling. The length adopted is the length of a medium sized coconut.

Therefore, Height of the blade = the maximum length of the coconut $=24 \mathrm{~cm}$

The depth of cut blades $=8 \mathrm{~cm}$.

Length of the gap between blade and main pipe is $=3 \mathrm{~cm}$

The cross section of the blade be a triangle of length $24 \mathrm{~cm}$.

Piston: The piston should have free movement inside the main pipe. Therefore the diameter of the piston is equal to $23.5 \mathrm{~cm}$ with close allowances.

By considering the maximum length of the coconut the stroke length adopted is $30 \mathrm{~cm}$

Area of the piston $=D^{2} / 4=23.5^{2} / 4=552.25 \mathrm{~cm}^{2}$

Pressure on piston $=\mathrm{F} / \mathrm{A}=600 / 552.25=1.08 \mathrm{Kg} / \mathrm{cm} 2$

Frame: Mild steel angles of 800 × 800 x $10 \mathrm{~mm}$ are used so that all the blade assemblies can occupy space with its fitting. The vertical height of the frame is fixed to $120 \mathrm{cms}$ so that operator will be in a comfortable position to operate the system.

Adams View is used to displays a dialog box that assists Design Engineer create a new modelling database or modify an existing one in which to save your models.

\section{RESULTS AND DISCUSSIONS}

The coconut dehusking machine was conceptually designed first using Solid works and analysed using ADAMS software. The amount of force with which the piston has to push the coconut is the force which is applied on the blades and piston. All these forces can be shown graphically by plotting time along $\mathrm{x}$-axis and force along $\mathrm{y}$-axis. The time is measured in seconds and force is measured in Newton

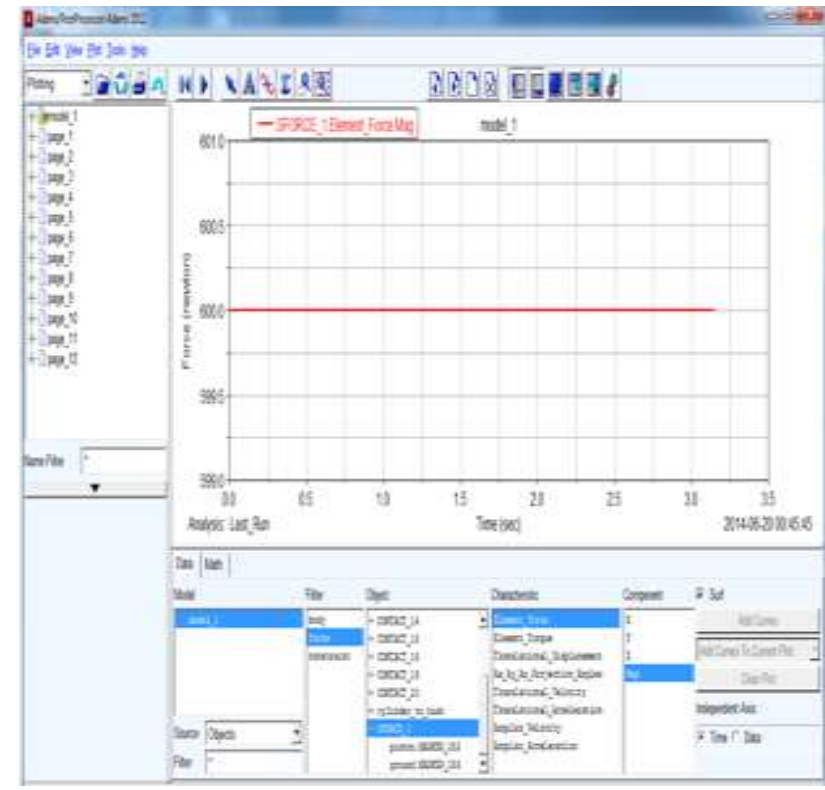

Fig 6 Force with which piston moves (N)

The above graph shows the force with which the piston moves over a period of time. As disused already previously the force on the piston should be $600 \mathrm{Kg}$. The force in the graph is showing that force on piston remains constant at all times during operation i.e. $600 \mathrm{Kg}$.

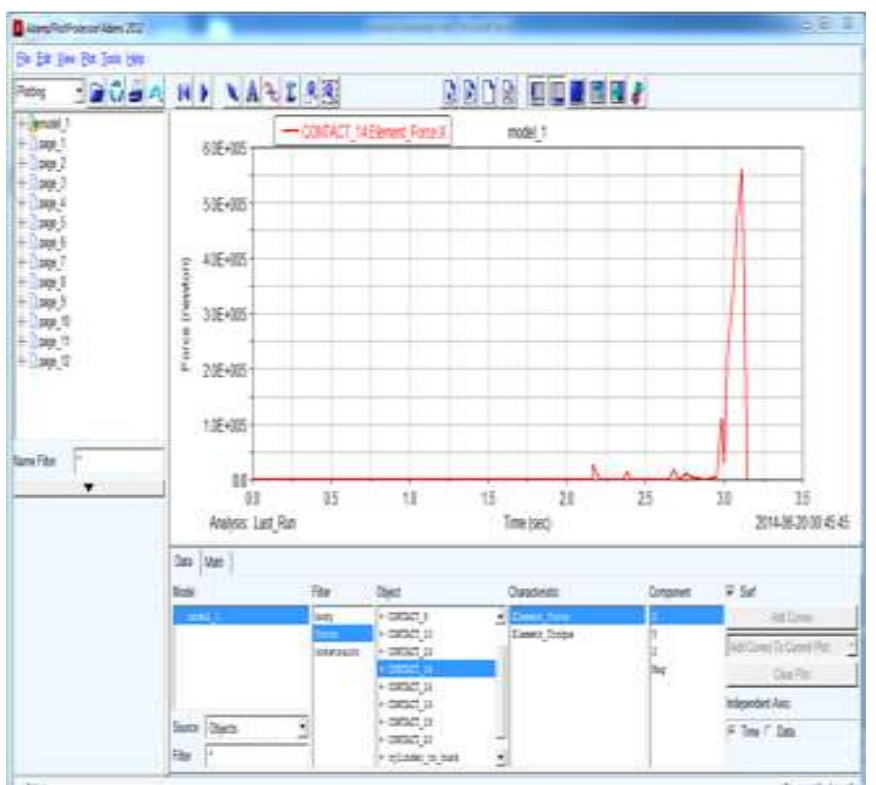

Fig 7 Force acting on the Coconut $(\mathrm{N})$

The graph above shows the force on coconut due to single blade and piston. Initially there is no force on the coconut and when the piston starts pushing the coconut the blade pierces into it and the force suddenly increases which can be clearly observed in the graph, force becomes zero when dehusking operation completes. 


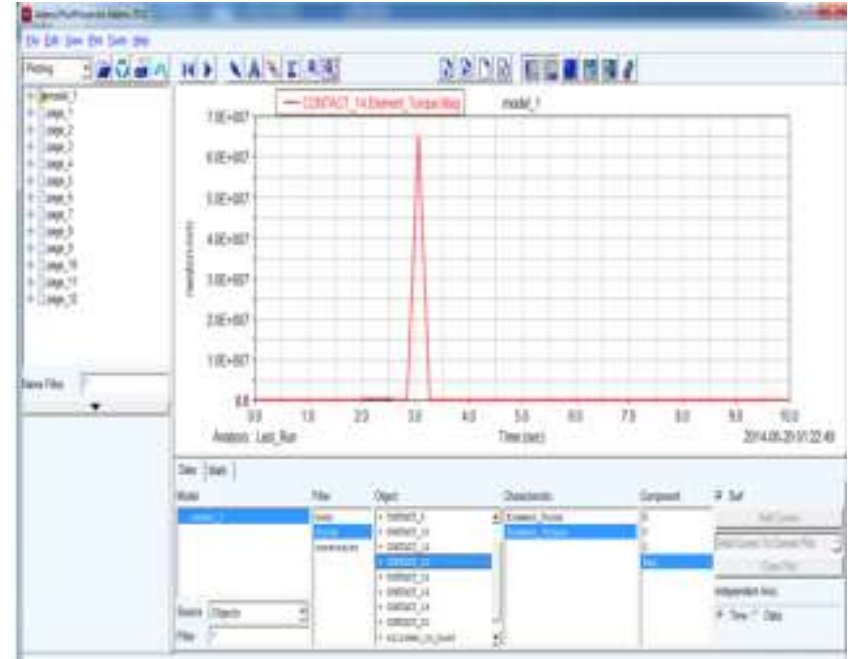

Fig 8 Torque acting on the Coconut(N-m)

The graph shown above represents the torque on the coconut. When the piston pushes coconut the torque increases suddenly as shown in graph. Once the coconut passes over the blades the torque reduces to zero.

\subsection{Position Analysis of Parts}

This section shows the positions of the objects or parts during the operation. The movement of objects causes the change in position with respect to their initial position. The position analysis can be shown in graphs by plotting time v/s distance that is time along $\mathrm{X}$-axis and length along $\mathrm{Y}$-axis. Where time is measured in seconds and length in millimetres.

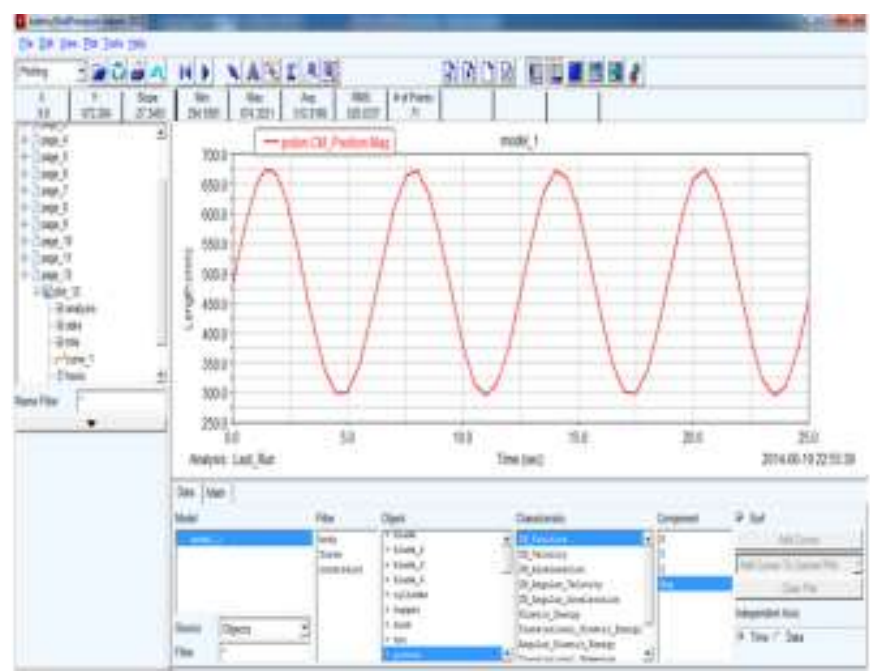

Fig 9 Position Analysis of Piston

This graph represents the movement of piston during the operation. The curve is periodic in nature because the piston has to move to and fro to ensure the repeated dehusking. The initial position of the piston is at $481 \mathrm{~mm}$ and it starts moving towards blade gradually. It takes 6 seconds from its initial position to reach the blade, after dehusking it takes 4 seconds to move up. This operation continues for the rest of the operation. The peaks at the bottom of the graph indicate that coconut is been dehusked and piston again travels up for second operation of dehusking.

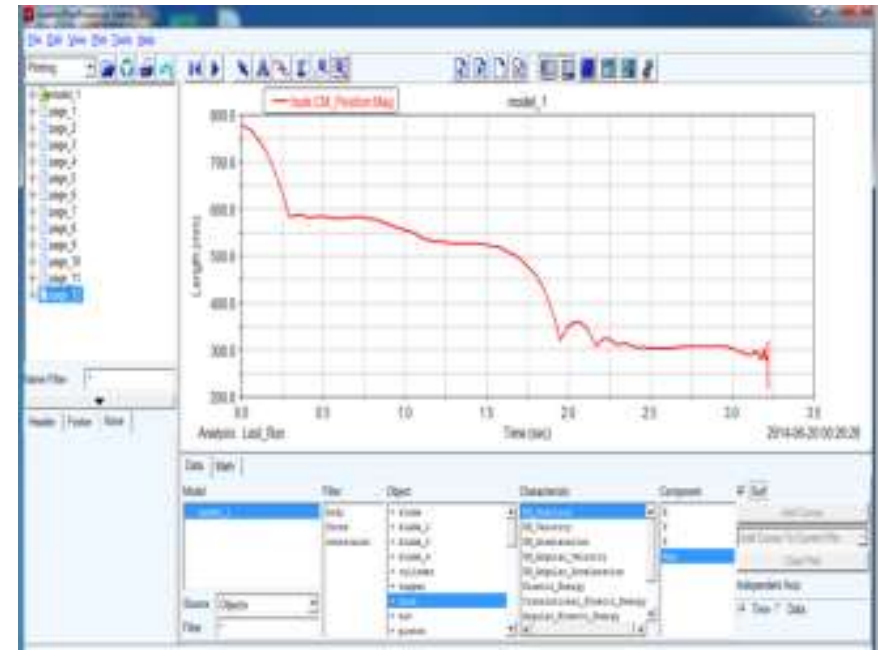

Fig 10 Position Analysis of Coconut

This graph shows the movement of Coconut during the operation, the coconut is fed into the hopper which is its initial position and it is at a height of $779 \mathrm{~mm}$; from there it gets positioned on the blades at time $\mathrm{t}=2$ seconds. Then curve becomes steady this period is the waiting period of coconut, at blades it waits for the piston to push it with the force of $600 \mathrm{Kg}(5.88 \mathrm{KN})$, then the dehusking operation completes. The dehusked coconut falls on the tray and becomes stationary.

Virtual simulation Design Analysis is generally adopted in product development in order to minimize the traditional reliance on testing of physical prototypes. It constitutes a major step towards solving the conflict of increase in cost and duration to enhance the competitiveness in the market by exploring alternative system design and obtaining instant feedback.

\section{CONCLUSION}

The virtual three dimensional CAD model is the reliable product development method. Virtual prototype to be developed is similar to physical product with humaninteraction. The virtual product can be observed, experienced by an design engineer or a user.In the present work the Coconut Dehusking Machine is modelled and the performance of that model is analyzed. The results are found satisfactory as per the design parameters, forces and torques these are explained and discussed. The expected parameters can be modified based on requirements and analysed for forces, torques and suitability.

The useful data thus generated can be used as an aid for the development of a customised product. It is hereby concluded that the Virtual Prototyping method, combined with flexible multi-body dynamic analysis is of great significance for predicting dynamic characteristics and for optimization of product during design stage. 


\section{ACKNOWLEDGEMENT}

I express my first and fore most panamas to his Holiness Ma.Gha.Cha. Channabasava Pattadevaru, Dr.Bheemanna Khandre Founder President, Er.Eshwar Khandre president SVE Society. The author acknowledge with heartful thanks to Professor, Malnad college of Engineering Dr.S.Mohankumar who made this Endeavour possible and also express our gratitude and indebtedness to Principal and Management of MCE, Hassan for providing us an opportunity to undergo research work successfully.

\section{REFERENCES}

[1] Dr. S. Mohan Kumar (2008) "Virtual Prototyping Technology - An Aided Design for Product development" International conference on Total Engineering, Analysis and Manufacturing Technologies at TEAMTECH -2008, 22-24 September 2008, at IISC, Bangalore.

[2] Design Data Hand Book for Mechanical Engineers,3rd edition by K.Mahadevan and K.Balaveera Reddy,

[3] Nagraja K.S,Manjunathprasad H.P,Aruna H.N,(201112) A project report on Design and fabrication of Coconut Dehusking Machine

[4] R.K. Singh and B. Subburaj, Market share of Tender nuts - AnEstimation -Indian Coconut Journal 8-10, Vol. XXXIV No. 5 September, 2003.

[5] Gopalkrishna M S, Prasanna S V, Ramesh Bhat H, "Design And Fabrication of Coconut Dehusker",sponsored by KSCST(Library Accession No.466)

[6] T.V Venkatesh Babu,Ahmed Sharief, Irfan Siddique,(1988-89) A project report on "Design of Coconut Dehusking Machine".

[7] Guo X. W., Z. X. Wang, L. Q. Wang, "Conceptual Design and Simulating Research Based on Virtual Prototyping Technology for Parallel Machine Tool(PMT)", Applied Mechanics and Materials vols. 10 - 12, 2008, pp 416-423, Trans

[8] B. N. Nwankwojike, O. Onuba, U. Ogbonna , "Development Of A Coconut Dehusking Machine For Rural Small Scale Farm Holders" International Journal Of Innovative Technology \& Creative Engineering Issn:2045-8711)Vol.2 No.3 Mar 2012Tech Publications. Switzerland.

[9] Rajashekar M, Ashok, Anita," Multibody Dynamic Analysis Of Mechanism For Health Exercise Vibrator" IJRET: International Journal of Research in Engineering and Technology Volume: 03 Special Issue: 03, p 550-553 May-2014, NCRIET-2014

[10] Rajashekar M , V K Heblikar S.Mohan Kumar," Simulation and Analysis of Low Cost Weeder", International Journal of Research in Engineering and Technology Volume: 03 Special Issue: 03, p 543-549 May-2014, NCRIET-2014 